\title{
Métodos de caracterización modal de guías de onda multinúcleo
}

\author{
David Benedicto, Maria Victoria Collados, Juan Carlos Martín, Jesús Atencia, Juan \\ Antonio Vallés \\ Tecnologías Ópticas Láser (TOL) \\ Instituto de Investigación en Ingeniería de Aragón (I3A) \\ Universidad de Zaragoza, Mariano Esquillor s/n, 50018, Zaragoza, Spain. \\ Tel.+34-876 5534 48, e-mail: dbenedicto@ unizar.es
}

\begin{abstract}
Las estructuras multinúcleo integradas son buenas candidatas para mejorar las prestaciones de los dispositivos ópticos activos [1]. Estamos trabajando en la caracterización de estas estructuras fabricadas mediante escritura por láser de femtosegundo [2]. Se presentan las dos técnicas de caracterización modal implementadas por nuestro grupo.
\end{abstract}

\section{Introducción}

El control sobre la composición modal es esencial para el diseño optimizado de estructuras integradas activas multinúcleo. En la literatura se encuentran dos técnicas experimentales de caracterización modal para este tipo de estructuras: (1) el registro con una cámara de las distribuciones transversales de potencia a la salida de la estructura y su tratamiento por ordenador mediante técnicas de imagen y (2) el uso de Elementos Ópticos Holográficos (EOHs) para separar espacialmente en tiempo real las componentes de cada modo.

\section{Caracterización por distribución de potencia}

El número de modos permitidos y la distribución transversal de potencia de cada uno son propiedades características de cada guía y de la longitud de onda de trabajo. Disponemos del software necesario para calcular todo ello, dada la distribución transversal de índice de refracción (RSoft CAD). En cambio, el reparto de la potencia total acoplada a la guía entre sus diferentes modos depende de cómo se excita esta.

La Figura 1 muestra el montaje experimental que permite variar la excitación de la guía y medir su distribución transversal de potencia. Su localización, dentro del sustrato en que ha sido grabada, es posible gracias a un microscopio. Este facilita también la optimización del enfrentamiento entre la entrada de la guía y la fibra óptica que le proporciona la luz. Para optimizar y asegurar la estabilidad del montaje, las posiciones de la fibra y la guía se regulan con microposicionadores controlables mediante piezoeléctricos. Cualquier desplazamiento lateral entre fibra y guía modifica obviamente la potencia total acoplada, pero también la proporción de potencia que porta cada modo. Como resultado, la distribución transversal de potencia cambia a medida que se descentra el enfrentamiento entre fibra y guía.

Junto al otro extremo de esta se coloca un objetivo de microscopio que forma en una cámara CCD (Bobcat 320 de Xenics) la imagen de la cara de salida de la guía. El aumento que proporciona el sistema óptico, ajustable mediante el desplazamiento del objetivo respecto a la salida de la guía, alcanza fácilmente un factor 200x. Teniendo en cuenta que el tamaño de los píxeles de la cámara es de $20 \mu \mathrm{m}$, puede disponerse de una resolución de hasta $0.1 \mu \mathrm{m}$ en la distribución transversal de potencia de salida de la guía. La proporción de la potencia total que porta cada modo se determina mediante ajuste de la imagen obtenida a una suma ponderada de las distribuciones transversales características de cada modo, previamente calculadas mediante el software mencionado.

\section{Caracterización mediante CGH.}

Otra forma de conocer la distribución modal a la salida de la guía es mediante el uso de hologramas generados por ordenador (CGH) [3]. Estos hologramas se pueden calcular y registrar digitalmente en un dispositivo modulador espacial de luz (SLM). Cuando se registra un holograma con la interferencia de una onda plana de referencia, $R, \mathrm{y}$ una onda objeto, $O_{n}$, formada por la distribución de amplitud compleja de uno de los modos, $n$, a la salida de la guía, la transmitancia del elemento holográfico, $t(x, y)$, es proporcional a la intensidad de dicha interferencia, 
$t(x, y)=\left[O_{n}(x, y)+R(x, y)\right]\left[O_{n}{ }^{*}(x, y)+\right.$

$\left.R^{*}(x, y)\right]=O_{n} O_{n}{ }^{*}+R R^{*}+O_{n} R^{*}+O_{n}{ }^{*} R$

Los dos últimos sumandos tienen información tanto de la amplitud como de la fase de ambas ondas. Si registramos digitalmente el cuarto sumando en un SLM de fase, cuando el SLM se ilumine con la distribución de potencia a la salida de guía, $\sum_{n} c_{n} O_{n}$, a la salida del SLM se tendrá una onda plana $c_{n} R$, en la dirección de la onda de referencia $R$, cuya intensidad es proporcional a la intensidad del modo $n$.

En el SLM puede registrarse la interferencia de cada uno de los modos con una onda de referencia $R$ inclinada con diferente ángulo para cada modo. De esta manera, a la salida del SLM podremos identificar la contribución de cada modo a través de las ondas difractadas, siendo su relación de intensidades proporcional a la de los diferentes modos a la salida de la guía. Para conseguir sobre el modulador la misma distribución de amplitud que se tiene a la salida de la guía, se ha incluido en el montaje un sistema $4 f$ formado por dos lentes (L1 y L2). El haz difractado por el elemento holográfico registrado en el SLM se propaga en una dirección diferente y su intensidad se recoge en un detector con ayuda de una lente (L3) (Figura 2).

\section{Conclusiones}

La caracterización modal de guías integradas multinúcleo mediante dos métodos experimentales diferentes permite contrastar su validez y contribuye al diseño optimizado de estas estructuras.

\section{REFERENCES}

[1]. ABEDIN K. S., FINI J. M., THIERRY T. F., SUPRADEEPA V. R., ZHU B., YAN M. F., BANSAL L., MONBERG E. M. y DIGIOVANNI D. J. Multicore erbium doped fiber amplifiers for space division multiplexing systems. $J$. of Lightwave Technol. 2014, 32(16), 2800-2808.

[2]. FERNANDEZ T. T., HARO-GONZÁLEZ P., SOTILLO B., HERNANDEZ M., JAQUE D., FERNANDEZ P., DOMINGO C., SIEGEL J. y SOLIS J. Ion migration assisted inscription of high refractive index contrast waveguides by femtosecond laser pulses in phosphate glass. Opt. Lett. 2013, 38(24), 5248-5251.

[3]. KAISE T., FLAMM D., SCHRÖTER S. y DUPARRÉ M. Complete modal decomposition for optical fibers using CGH-based correlation filters. Opt. Express. 2009, 17(11), 9347-9356.

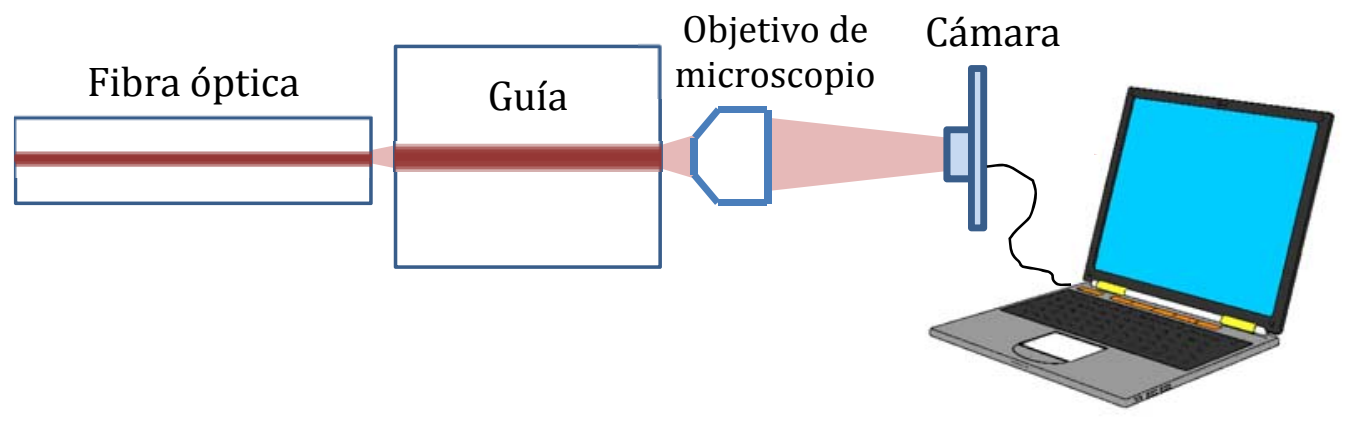

Fig. 1 Montaje experimental para medir la distribución transversal de potencia a la salida de una guía

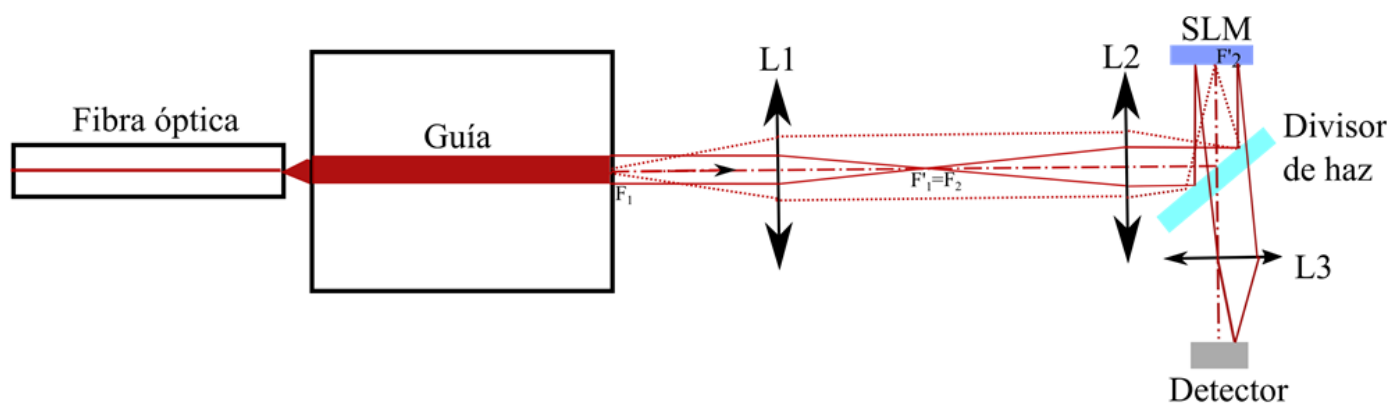

Fig. 2: Montaje para el reconocimiento de modos con un elemento óptico holográfico 